\title{
MRT bei neurodegenerativen Erkrankungen
}

Horst Urbach, Karl Egger

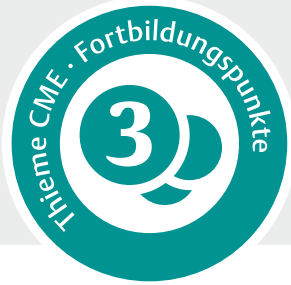

\section{Neurodegenerative Erkrankungen nehmen in einer älter werdenden Gesellschaft stark zu. Bei klinischem Verdacht lassen sich diese Erkrankungen häufig anhand krankheitsspezifischer Atrophiemuster diagnostizieren. Die Erkennung wird er- leichtert, wenn der Datensatz des Patienten mit einer Gruppe „alters- und ge- schlechtsgematchter" Kontrollen verglichen und Voxel bzw. Regionen, die sich unterscheiden, hervorgehoben werden.}

$\begin{array}{ll}\text { ABKÜRZUnGEN } \\ \text { AD } & \text { Alzheimer-Demenz } \\ \text { ERICA } & \text { „entorhinal cortex atrophy“ } \\ \text { FTD } & \text { frontotemporale Demenz } \\ \text { MSAC } & \text { Multisystematrophie vom zerebellären } \\ & \text { Prädominanztyp } \\ \text { MSAP } & \text { Multisystematrophie vom Parkinson- } \\ & \text { Prädominanztyp } \\ \text { MTA } & \text { mesiale temporale Atrophie } \\ \text { PD } & \text { Parkinson' Disease, Parkinson- } \\ & \text { Krankheit } \\ \text { PSP } & \text { progressive supranukleäre Paralyse } \\ \text { PSP-RS } & \text { Richardson Steele } \\ \text { PSP-CBD } & \text { kortikobasale Degeneration } \\ \text { NPH } & \text { „normal pressure hydrocephalus“ } \\ \text { ASL } & \text { „arterial spin labeling“ } \\ \text { LBD } & \text { Lewy-Body-Demenz } \\ & \end{array}$

\section{Demenzerkrankungen}

\section{Klinische Definition}

Demenzerkrankungen sind definiert durch den im Verlauf zunehmenden Abbau und Verlust kognitiver Funktionen und Alltagskompetenzen. Es kommt u. a. zu Beeinträchtigungen der zeitlich-örtlichen Orientierung, der Kommunikationsfähigkeit, der autobiografischen Identität und von Persönlichkeitsmerkmalen bis hin zur vollständigen Hilflosigkeit und Abhängigkeit von der Umwelt [1].

Bei anderen Erkrankungen wie dem Morbus Parkinson oder den atypischen Parkinson-Syndromen ist zwar die Bewegungsstörung das Leitsymptom - jedoch gehen auch diese Erkrankungen in späteren Stadien häufig mit einer Demenz einher.

\section{Epidemiologie}

Die Anzahl von Demenzkranken in Deutschland wird auf ca. 1,2 Millionen geschätzt, leichte Demenzstadien werden möglicherweise unterschätzt [2]. Die Prävalenz ist stark altersabhängig, Frauen erkranken häufiger als Männer [1].

\section{Pathophysiologie}

Vereinfacht dargestellt entstehen die sog. primären Demenzerkrankungen Alzheimer-Demenz (AD), frontotemporale Demenz (FTD), Lewy-Körperchen-Demenz (LBD), Morbus Parkinson mit Demenz (PDD) und atypische Parkinson-Syndrome (APS) durch die Ablagerung bzw. den ungenügenden Abtransport von Proteinen im Hirnparenchym [3].

\section{Merke}

Bei der ADhandelt es sich um im Neuropil abgelagerte Amyloid- $\beta$ - und intraneuronale Tau-Proteine, bei der FTD um Tau- und andere Proteine, beim Morbus Parkinson (PD) um intraneuronales $\alpha$-Synuclein.

Zu den atypischen Parkinson-Syndromen (APS) zählen sowohl $\alpha$-Synucleinopathien (LBD, MSAc, MSAp) als auch Tau-Erkrankungen wie die kortikobasale Degeneration (CBD) und die progressive supranukleäre Paralyse (PSP) einschließlich ihrer Varianten. Die Proteinaggregate werden z. T. lange Zeit vor Beginn der klinischen Symptome abgelagert und bedingen im Verlauf Hypometabolismus, Hypoperfusion und Atrophie des Gewebes, die je nach Erkrankung in bestimmten Regionen des Gehirns beginnen [4]. Erschwert wird die pathophysiologische Einteilung jedoch durch zusätzlich auftretende Proteinopathie Überlappungen. Aus Gründen der Übersichtlichkeit sind die o.g. Erkrankungen einschließlich ihrer wesentlichen MRT-Befunde in - Tab. 1 zusammengefasst. 
-Tab. 1 Primäre Demenzerkrankungen bzw. mit Demenz einhergehende neurodegenerative Erkrankungen.

\begin{tabular}{|c|c|c|c|c|}
\hline Demenz & Varianten & $\begin{array}{l}\text { relative } \\
\text { Häufigkeit }\end{array}$ & Klinik & MRT \\
\hline \multirow{2}{*}{$\begin{array}{l}\text { Morbus Alzheimer } \\
\text { [24] }\end{array}$} & typisch (90\%) & \multirow[t]{2}{*}{$50-70 \%$} & \multirow{2}{*}{$\begin{array}{l}\text { - Störung des episodischen Kurzzeit- } \\
\text { gedächtnisses } \\
\text { - andere kognitive Störung, kein Delir } \\
\text { - durch Gedächtnisstörung bedingte } \\
\text { Störung der Alltagsfähigkeit } \\
\text { - seit mehr als } 6 \text { Monaten bestehend }\end{array}$} & \multirow{2}{*}{$\begin{array}{l}\text { temporomesiale und parietale Atrophie, } \\
\text { vor allem des entorhinalen Kortex und } \\
\text { des Präcuneus }\end{array}$} \\
\hline & $\begin{array}{l}\text { atypisch (10\%) } \\
\text { - posteriore korti- } \\
\text { kale Atrophie } \\
\text { (PCA) } \\
\text { - logopenische } \\
\text { Aphasie } \\
\text { - frontale Variante }\end{array}$ & & & \\
\hline vaskuläre Demenz & & $15-25 \%$ & $\begin{array}{l}\text { - kognitiver Abbau mit häufig abrup- } \\
\text { tem Beginn } \\
\text { - Schlaganfälle in der Anamnese } \\
\text { - fokale neurologische Defizite }\end{array}$ & $\begin{array}{l}\text { - ausgeprägte Mikroangiopathie } \\
\text { " „strategische“ Infarkte } \\
\text { " Makroangiopathie mit Beteiligung } \\
\text { beider Thalami und/oder temporo- } \\
\text { mesialer Strukturen }\end{array}$ \\
\hline \multirow[t]{2}{*}{$\begin{array}{l}\text { frontotemporale } \\
\text { Lobärdegeneration }\end{array}$} & $\begin{array}{l}\text { behaviorale Variante } \\
\text { (bvFTLD) [27] }\end{array}$ & \multirow[t]{2}{*}{$20 \%$} & $\begin{array}{l}\text { - Verhaltensauffälligkeiten, Wesens- } \\
\text { änderung } \\
\text { - bei Befall der dominanten } \\
\text { Hemisphäre Aphasie }\end{array}$ & $\begin{array}{l}\text { - ein- oder beidseitig frontotemporale } \\
\text { Atrophie ( } \triangleright \text { Abb. } 4 \text { ) } \\
\text { - Beteiligung der mesiotemporalen } \\
\text { Strukturen möglich }\end{array}$ \\
\hline & $\begin{array}{l}\text { primär progressive } \\
\text { Aphasie (PPA) [28] } \\
\text { - } \text { nicht flüssige PPA } \\
\text { - } \text { semantische PPA } \\
\text { - logopenische PPA }\end{array}$ & & Leitsymptom Aphasie & $\begin{array}{l}\text { Atrophie im Bereich der sprachrelevan- } \\
\text { ten Areale; bei der semantischen PPA im } \\
\text { vorderen Temporallappen bevorzugt } \\
\text { links ( } \triangleright \text { Abb. } 5 \text { a-d), bei der logopeni- } \\
\text { schen PPA im Bereich des frontalen } \\
\text { Operculums }\end{array}$ \\
\hline $\begin{array}{l}\text { Demenz mit Lewy- } \\
\text { Körperchen (DLB) } \\
\text { [33] }\end{array}$ & & $5 \%$ & $\begin{array}{l}\text { - Funktionseinschränkungen im } \\
\text { Alltag bei zu Beginn oft noch gut } \\
\text { erhaltenem Gedächtnis } \\
\text { - Fluktuation von Aufmerksamkeit } \\
\text { und Wachheit } \\
\text { - Parkinson-Syndrom in } 25-50 \% \text { der } \\
\text { Fälle bei Erkrankungsbeginn } \\
\text { - visuelle Halluzinationen } \\
\text { - Neuroleptika-Überempfindlichkeit }\end{array}$ & $\begin{array}{l}\text { - im fortgeschrittenen Stadium Atro- } \\
\text { phien im Parietal- und Okzipitallap- } \\
\text { pen möglich } \\
\text { - hippokampale Atrophie seltener }\end{array}$ \\
\hline Parkinson-Demenz & & & $\begin{array}{l}\text { Demenz beginnt i. d. R. > } 1 \text { Jahr nach } \\
\text { der Parkinson-Symptomatik }\end{array}$ & kein pathognomonischer Befund \\
\hline \multirow[t]{5}{*}{$\begin{array}{l}\text { atypische Parkinson- } \\
\text { Syndrome }\end{array}$} & DLB & & s.o. & $\begin{array}{l}\text { - kein pathognomonischer Befund } \\
\text { - Signalerhöhung der mittleren Klein- } \\
\text { hirnstiele, „hot-cross-bun“-Zeichen } \\
\text { - asymmetrische Volumenminderung } \\
\text { frontotemporoparietal mit Beto- } \\
\text { nung der Zentralregion }\end{array}$ \\
\hline & MSAC & & & $\begin{array}{l}\text { Pons- und Zerebellum-Atrophie } \\
\text { ( } \triangleright \text { Abb.6a-c) }\end{array}$ \\
\hline & MSAp & & & $\begin{array}{l}\text { Atrophie speziell der posterioren Puta- } \\
\text { mina ( } \triangleright \text { Abb. } 6 \text { d-e) }\end{array}$ \\
\hline & CBD & & & $\begin{array}{l}\text { meist seitenasymmetrische Atrophie } \\
\text { zentral betont ( } \triangleright \mathbf{A b b} \mathbf{6} \mathbf{g})\end{array}$ \\
\hline & PSP-Varianten [32] & & & \\
\hline
\end{tabular}


- Tab. 1 (Fortsetzung)

\begin{tabular}{|c|c|c|c|c|}
\hline Demenz & Varianten & $\begin{array}{l}\text { relative } \\
\text { Häufigkeit }\end{array}$ & Klinik & MRT \\
\hline & PSP-RS & & & $\begin{array}{l}\text { Hirnstamm: } \\
\text { " Midbrain-Pons-Ratio<0,52 } \\
\text { " Kolibri-Zeichen } \\
\text { - Mickey-Mouse-Zeichen } \\
\text { - Atrophie des Tegmentum pontis } \\
\text { und der oberen Kleinhirnstiele } \\
\text { Großhirn: im fortgeschrittenen } \\
\text { Stadium frontale bis parietale } \\
\text { Atrophie ( } \text { Abb. } 7 \text { a-d) }\end{array}$ \\
\hline & $\begin{array}{l}\text { - PSP-Parkinsonism } \\
\text { - PSP pure akinesia } \\
\text { - PSP non-fluent } \\
\text { aphasia } \\
\text { - PSP-CBD }\end{array}$ & & & $\begin{array}{l}\text { - Großhirn: im fortgeschrittenen Sta- } \\
\text { dium frontale bis parietale Atrophie } \\
\text { - bei PSP-CBD akzentuierte, teils } \\
\text { asymmetrische zentrale Atrophie }\end{array}$ \\
\hline & $\begin{array}{l}\text { - PSP predominant } \\
\text { cerebellar ataxia }\end{array}$ & & & $\begin{array}{l}\text { Mittelhirnatrophie und zerebelläre } \\
\text { Atrophie bei relativ unauffälligem Pons } \\
\text { (wichtig für Differenzialdiagnose MSAc) }\end{array}$ \\
\hline
\end{tabular}

\section{Klinische Verteilung}

Nach klinischen Kriterien sind ca. 50-70\% der Demenzerkrankten der Alzheimer-Demenz und ca. 20\% der vaskulären Demenz zuzuordnen $[5,6]$. Mischdemenzen sind hier eingerechnet. Etwa $20 \%$ der Personen mit einer Demenz vor dem 65. Lebensjahr haben vermutlich eine FTD [7]. Die Anzahl der an Morbus Parkinson Erkrankten in Deutschland beträgt ca. 100000 , die Punktprävalenz von Morbus Parkinson plus Demenz (PDD) zwischen 20 und 40\%. Langzeitstudien an sehr kleinen Fallzahlen zeigen einen Anstieg der Prävalenz der Parkinson-Demenz bis zu $80 \%$ bei einem Krankheitsverlauf von 12 bzw. 20 Jahren [8]. Eine systematische Literaturrecherche ergab für die LBD eine Prävalenz von $0-5 \%$ in der Allgemeinbevölkerung und von $0-30 \%$ bei Demenzkranken [9], wobei eine klare Abgrenzung zwischen der PDD und der LBD lediglich durch den zeitlichen Verlauf ermöglicht wird (bei der LBD tritt die Demenz deutlich früher auf).

\section{Merke}

Alle neurodegenerativen Demenzerkrankungen (AD, FTD, PDD, LBD, APS) sind progressive Erkrankungen mit sehr variablen Verläufen über mehrere Jahre.

Die FTD sowie atypische AD-Formen wie die posteriore kortikale Atrophie (PCA) zeigen im Mittelwert einen früheren Erkrankungsbeginn (im Mittel < 65 Jahre) als die übrigen genannten Erkrankungen [7]. Bei der vaskulären Demenz, die nicht selten in Kombination mit der ADauftritt (=vaskuläre Mischdemenz), kommen auch stufenförmige Verläufe mit teilweise langen Pha- sen ohne Progredienz und Phasen leichter Besserung vor.

\section{Bildgebung}

Der bildgebenden Untersuchung kommt bei der Diagnostik neurodegenerativer Erkrankungen 2 Funktionen zu: Sie soll behandelbare Ursachen aufdecken (z. B. Tumor, subdurales Hämatom, Normaldruckhydrozephalus) und zur ätiologischen Differenzierung primärer Demenzerkrankungen beitragen [1].

\section{NPH-Aspekt}

Behandelbare Erkrankungen wie Tumor und Subduralhämatom liegen in deutlich weniger als $5 \%$ der Fälle vor $[10,11]$. Ein nicht okklusiver Hydrozephalus (u.a. Normal Pressure Hydrocephalus-NPH) wird jedoch häufiger gefunden. Es wurde jahrelang davon ausgegangen, dass die Symptome Gangstörung und Harninkontinenz innerhalb der Hakim-Trias prädiktiver sind für einen NPH als das Symptom kognitiver Abbau. In neueren Arbeiten waren NPH-typische Ventrikelerweiterungen bei Demenzpatienten jedoch häufiger als bei altersgematchten Kontrollen.

Merke

Ein weiterer wichtiger Aspekt in Bezug auf die Bildgebung ist die Erkenntnis, dass das NPH-Bild ( = NPHAspekt) bereits länger als 3 Jahre vor Beginn der klinischen Symptomatik vorliegen kann [12]. 
Zuverlässigste bildmorphologische Diagnosekriterien sind dabei das DESH-Zeichen und der Corpus-callosum-Winkel:

- Beim DESH-Zeichen (DESH = „disproportional enlarged subarachnoid space hydrocephalus") besteht eine Diskrepanz zwischen den ballonierten inneren Liquorräumen bzw. der erweiterten Sylvischen Fissur und den enggestellten hochparietalen äußeren Liquorräumen.

- Der Corpus-callosum-Winkel (gemessen auf Höhe der Commissura posterior) beträgt beim NPH-Aspekt unter $90^{\circ}[13]$ ( $\mathbf{A b b}$. 1$)$.

Für die Diagnose des NPH-Aspekts wird an der Freiburger Klinik für Neuroradiologie zusätzlich zur visuellen Beurteilung ein automatischer „machine-learning“-Algorithmus verwendet. Erste noch unveröffentlichte Daten zeigen eine äußerst hohe Zuverlässigkeit der automatischen SVM-Analyse (SVM = Support-Vector-Machine) in Bezug auf die MRT-morphologische Diagnose „NPH-Aspekt“ mit einer Sensitivität und Spezifität von über $90 \%$, basierend auf einem WahrscheinlichkeitsThreshold von $50 \%$ (Egger et al., unveröffentlichte Daten) ( $\boldsymbol{A} \mathbf{A b b} \mathbf{1} \mathbf{d}$ ). Liegt ein NPH-Aspekt vor, ist jedoch zu beachten, dass die visuelle, wie auch automatische volumetrische Beurteilung bezüglich spezifischer Hirnatrophiemuster nicht mehr zuverlässig durchgeführt werden kann.

\section{Neurodegenerations-MRT}

Sequenzen

Folgende MRT-Sequenzen halten wir für unverzichtbar:

- T1w 3-D-Sequenz mit isotropen, etwa $1 \mathrm{~mm}^{3}$ großen Voxeln, bevorzugt in sagittaler Schichtführung

- FLAIR-Sequenz, wobei diese als 3-D-Sequenz mit isotropen, etwa $1 \mathrm{~mm}^{3}$ großen Voxeln oder als 2-DSequenz mit Schichtdicken von $3 \mathrm{~mm}$ aufgenommen werden sollte

- axiale SWI oder T2* -Sequenzen mit 2 bis maximal $5 \mathrm{~mm}$ dicken Schichten

- axiale DWI-Sequenz mit maximal 5 mm dicken Schichten

- axiale T2w Sequenz mit maximal 5 mm dicken Schichten

Die T1w 3-D-Sequenz wird aus Messzeitgründen sagittal akquiriert. Dabei wird durch die sagittale Schichtführung auch die Abdeckung (FOV) optimiert. Für eine standardisierte visuelle Beurteilung sollten jedoch zusätzlich koronare Schichten (bevorzugt in temporaler Kippung bzw. parallel zum Hirnstamm) zur Beurteilung der medialen (mesialen) Anteile der Temporallappen und der Frontallappen sowie axiale Schichten zur Beurteilung der Parietallappen aus dem 3-D-Datensatz rekonstruiert werden. Sagittale Schichtaufnahmen in der Mittellinie sind zur Beurteilung des Hirnstamms, paramediane sagittale Aufnahmen zur Beurteilung des Präcuneus hilfreich $[14,15]$.

Visuelle und semiquantitative Beurteilung

Für die visuelle und semiquantitative Beurteilung kommen der MTA-Score (Scheltens-Score), der ERICAScore, der Koedam-Score, die Pons-Midbrain-Ratio und eine volumetrische Analyse infrage ( $>$ Tab. 2).

Der sog. MTA-Score wird auf koronaren Schnitten bestimmt [14]. Dabei beurteilt man den Hippocampus optimalerweise in temporaler Kippung, wobei man auf Höhe des Hippocampuskopfes die Weite der Fissura choroidea, die Weite des Temporalhorns sowie die Höhe des Hippocampus berücksichtigt. Im Score bedeuten 0 Punkte keine und 4 Punkte das Vollbild einer hippokampalen Atrophie. Bei einem Score von 3 erscheint der Hippocampus im Seitenvergleich höhengemindert, bei einem Score von 4 ist diese Höhenminderung visuell eindeutig. Das Alter fungiert als Kovariable: Bei Personen unter 75 Jahren gilt ein Score von $\geq 2$, bei Patienten $\geq 75$ Jahren ein Score $\geq 3$ als pathologisch. Beide Seiten werden addiert und die Summe durch 2 geteilt. Die Intrarater-Variabilität wird mit 0,8 angegeben [16].

Beim ERICA-Score beurteilt man die Atrophie im entorhinalen Kortex, weil die Tau-Ablagerungen bei der Alzheimer-Demenz nicht im Hippocampus, sondern im entorhinalen Kortex, d. h. im vorderen Gyrus parahippocampalis, beginnen [17]. Enkirch und Mitarbeitern haben hierfür eine Skala von 0 bis 3 Punkten eingeführt, wobei das Stadium 1 durch einen erweiterten Sulcus collateralis und die Stadien 2 und 3 u. a. durch das sog. „tentorial cleft sign“, also einen sichtbaren Spalt zwischen entorhinalem Kortex und dem Tentorium cerebelli, charakterisiert sind [18].

Die parietale Atrophie wird im Koedam-Score auf einer Skala von 0 bis 3 beurteilt, auf der 0 Punkte keine und 3 Punkte das Vollbild einer parietalen Atrophie mit einer deutlichen Erweiterung des Ramus marginalis des Sulcus cinguli (Sulcus marginalis) und des Sulcus parietooccipitalis auf den parasagittalen Bildern bzw. eine Erweiterung der intraparietalen Sulci auf den axialen bzw. koronaren Bildern bedeuten [15] ( Abb.2, -Abb. 3).

Die Mittelhirn- und/oder Pons-Atrophie wird auf sagittalen T1w Aufnahmen in der Mittellinie erfasst, indem das Mittelhirn und der Pons mittels Ellipsen planimetriert und die kürzeren Durchmesser zueinander in Bezug gesetzt werden (Midbrain-Pons-Ratio, > Abb.4c, - Abb.7b) [19]. Aufwendiger ist die Berechnung des MR-Parkinson-Index, bei dem zusätzlich der Durchmesser der bei PSP normalen mittleren Kleinhirnstiele auf paramedianen Sagittalaufnahmen und der Durchmes- 

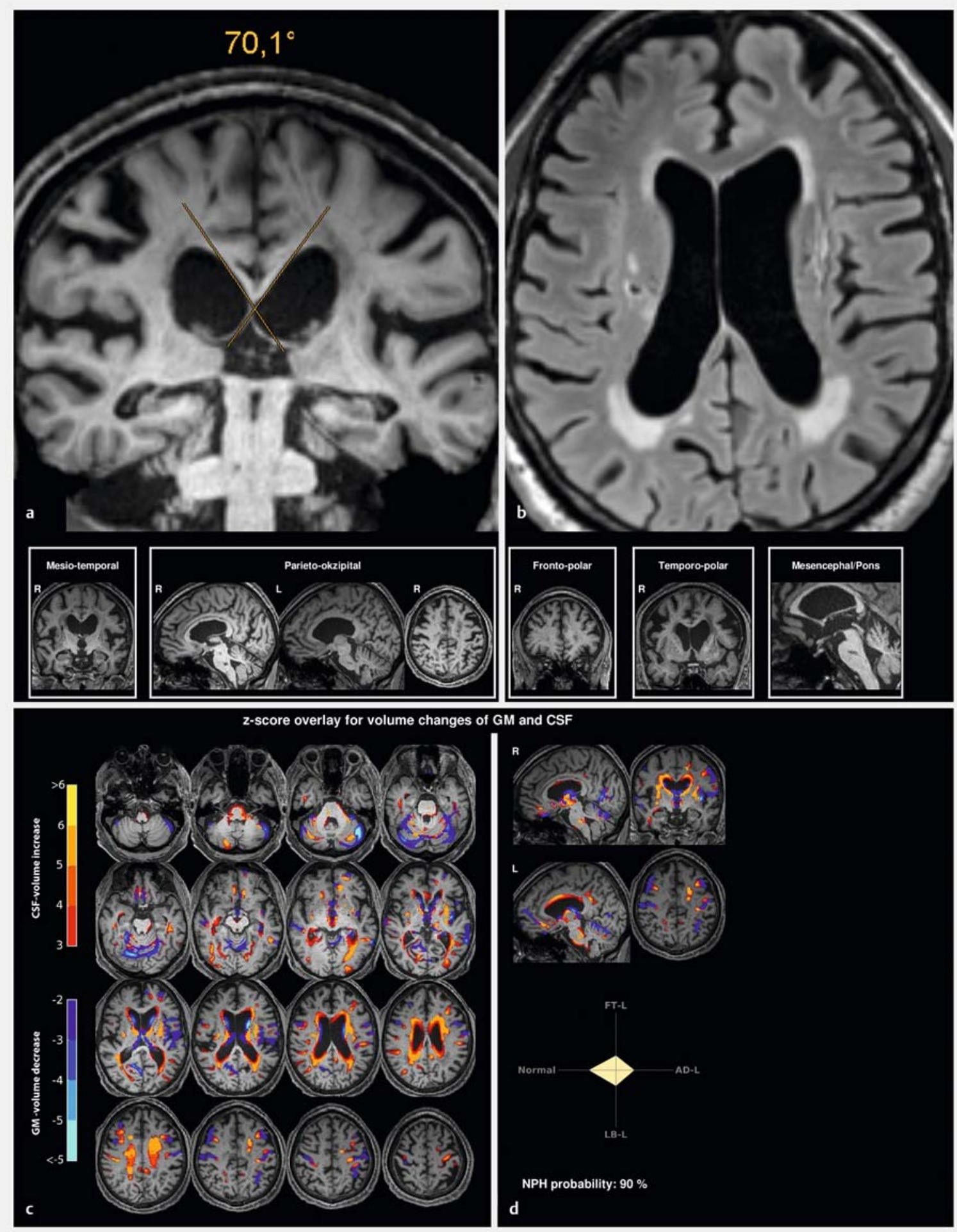

Abb. 1 73-jähriger Mann mit spastisch-ataktischer Gangstörung, Differenzialdiagnose MSAc. a Die inneren Liquorräume und die Sylvische Fissur sind erweitert, der Corpus-callosum-Winkel auf Höhe der Commissura posterior beträgt $70^{\circ}$. Die hochparietalen äußeren Liquorräume sind im Verhältnis zu den inneren Liquorräumen bzw. der Sylvischen Fissur enggestellt (= positives DESH-Zeichen). b Im FLAIR-Bild zeigen sich Hyperintensitäten um die Vorder- und Hinterhörner der Seitenventrikel. c Die volumetrische Analyse zeigt ebenfalls eine deutliche Zunahme des Volumens der inneren Liquorräume (in Rot- bis Gelbtönen). d Die auf der Basis einer Support-Vektor-Maschine ermittelte Wahrscheinlichkeit eines NPH-Aspekts liegt bei $90 \%$. Nach nochmaliger klinischer Testung bestätigte sich der Verdacht auf einen NPH, und die durchgeführte ventrikuloperitoneale Shuntanlage brachte eine eindeutige klinische Besserung. 
Tab.2 Visuelle und planimetrische Atrophie-Scores.

\begin{tabular}{|c|c|c|}
\hline Score & MRT & Auswertung \\
\hline MTA & 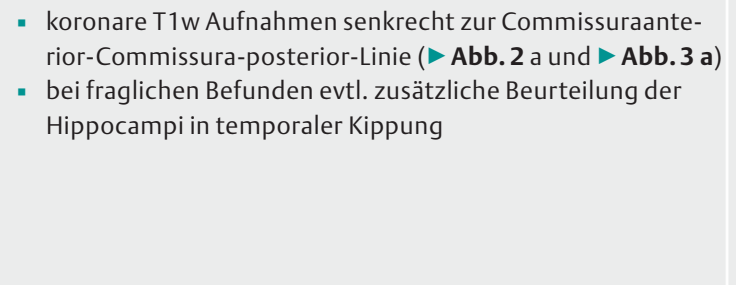 & $\begin{array}{l}0=\text { normal } \\
1=\text { erweiterte Fissura choroidea } \uparrow \\
2=\text { erweiterte Fissura choroidea } \uparrow \uparrow \text {, weites Unterhorn } \uparrow, \\
\text { Höhe der Hippocampusformation } \downarrow \\
3=\text { erweiterte Fissura choroidea } \uparrow \uparrow \uparrow \text {, weites Unterhorn } \uparrow \uparrow, \\
\text { Höhe der Hippocampusformation } \downarrow \downarrow \\
4=\text { erweiterte Fissura choroidea } \uparrow \uparrow \uparrow \text {, weites Unterhorn } \\
\uparrow \uparrow \uparrow, \text { Höhe der Hippocampusformation } \downarrow \downarrow \downarrow\end{array}$ \\
\hline Koedam & $\begin{array}{l}\text { T1w Aufnahmen } \\
\text { - sagittal: weiter R.marginalis sulci cinguli und weiter R.mar- } \\
\text { ginalis sulci parietooccipitalis, Präcuneus-Atrophie } \\
\text { " axial, koronar: weite Pars marginalis cinguli und weite intra- } \\
\text { parietale Furchen }\end{array}$ & $\begin{array}{l}0=\text { normal } \\
1=\text { leichte Atrophe } \\
2=\text { mäßige Atrophie } \\
3=\text { schwere Atrophie }\end{array}$ \\
\hline ERICA & koronare T1w Aufnahmen senkrecht zum Hirnstamm & $\begin{array}{l}0=\text { normal } \\
1=\text { erweiterter Sulcus collateralis } \\
2=\text { erweiterter Sulcus collateralis }+ \text { Tentorium-Cleft-Zeichen = } \\
\text { Liquorspalt zwischen entorhinalem Kortex und Tentorium } \\
3=\text { erweiterter Sulcus collateralis + deutliches Tentorium- } \\
\text { Cleft-Zeichen }\end{array}$ \\
\hline $\begin{array}{l}\text { Midbrain-Pons- } \\
\text { Ratio }\end{array}$ & $\begin{array}{l}\text { sagittale T1w Aufnahmen: Verhältnis der kurzen Durchmesser } \\
\text { der im Mittelhirn und Pons eingepassten elliptischen Flächen } \\
\text { ( } \triangleright \text { Abb.4 c, } \text { A Abb. } 7 \text { b) }\end{array}$ & PSPvs. Gesunde $<0,52$ \\
\hline $\begin{array}{l}\text { MR-Parkinson- } \\
\text { Index }\end{array}$ & $\begin{array}{l}\text { - sagittale T1w Aufnahmen: Pons-Fläche, Mittelhirn-Fläche, } \\
\text { mittlerer Kleinhirnstiel-Durchmesser } \\
\text { - koronare T1w Aufnahmen: oberer Kleinhirnstiel-Durchmes- } \\
\text { ser } \\
\text { - Berechnung: Pons-Fläche/Mittelhirn-Flächex (mittlerer } \\
\text { Kleinhirnstiel-Durchmesser/oberer Kleinhirnstiel-Durch- } \\
\text { messer) }\end{array}$ & PSP vs. Gesunde > 13,58 \\
\hline
\end{tabular}

ser der bei der PSP atrophen oberen Kleinhirnstiele auf parakoronaren Aufnahmen gemessen und zueinander in Bezug gesetzt werden [20].

Die Altersabhängigkeit der Atrophie erschwert eine verlässliche qualitative Beurteilung. Die Grenze von 75 Jahren für die mesiale temporale Atrophie (MTA) wurde eher empirisch gewählt und wird den unterschiedlichen Krankheitsvarianten und -verläufen kaum gerecht. Vergleicht man jedoch voxelweise oder in ausgewählten Regionen das Gehirn des Patienten mit einer Gruppe alters- und geschlechtsgleicher Gesunder, ist eine volumetrische Analyse möglich, d. h. die Volumenunterschiede lassen sich quantitativ erfassen:

- Das Gehirn des Patienten wird dazu in einen Standardraum transformiert und in die Gewebeklassen graue Substanz, weiße Substanz und Liquor segmentiert.

- Um das Volumen einer bestimmten anatomischen Struktur (z. B. des Nucleus caudatus) zu bestimmen, verwendet man sog. probabilistische digitale Hirnatlanten, z. B. den LPBA40-Atlas des Laboratory of Neuroimaging (http://www.loni.ucla.edu/Atlases/), den Shattuck-Atlas [21] oder den Jülich-Atlas, der die zytoarchitektonischen Felder kartiert (http://jubrain.fz-juelich.de).

- Multipliziert man die entsprechende Maske (z. B. Nucleus-caudatus-Maske) aus einem probabilistischen Hirnatlas mit dem normalisierten Bild des Patienten und summiert die im Patienten vorhandenen Voxel innerhalb der segmentierten Region, erhält man das Volumen des individuellen Nucleus caudatus.

- Nimmt man dann den Mittelwert inkl. der Standardabweichung aus einer ausreichend großen Vergleichsgruppe alters- und geschlechtsgematchter Gesunder, kann man das individuelle Patientenvolumen im Vergleich dazu als Zahl z. B. mittels Z-ScoreBerechnung darstellen. Diese Zahl stellt den Abstand der individuellen Messgröße vom Mittelwert aller Messwerte der Vergleichsgruppe in Standardabweichungen dar. Somit entspricht ein Wert von $z=1$ genau einer Standardabweichung.

Für ein an der Klinik für Neuroradiologie Freiburg entwickeltes vollautomatisches Volumetrie-Tool wurde als Grenzwert („threshold“) für „abnormal“ der wissenschaftlich gängige Wert von 2 Standardabweichungen 
$(z=2)$ definiert. Somit können z. B. regionale Volumenabnahmen in der Hirnrinde bzw. Volumenzunahmen in den äußeren und inneren Liquorräumen dem z-Wert entsprechend farblich codiert auf das individuelle Gehirn überlagert werden (s.Abbildungen).

Zusätzlich wird das individuelle Volumen mit unterschiedlichen Gruppen von gesunden Personen und Patienten mit typischem Atrophiemuster bzw. NPH-Aspekt mittels „machine-learning“-Algorithmen verglichen und die Wahrscheinlichkeit in Bezug auf die Zugehörigkeit zu einer dieser Gruppe berechnet (s.Abbildungen außer $\mathbf{A}$ Ab.6). In ersten Studien wurde die automatische Erkennung von Atrophien mittels ROCAnalyse (AUC bis zu 0,95) im Vergleich zum visuellen Experten-Reading als sehr zuverlässig beschrieben. Atrophien, die anhand der Erweiterung der äußeren Liquorräume erkannt wurden, zeigten tendenziell bessere Werte, wenngleich dadurch die klinische Beurteilung keinesfalls ersetzt werden kann [22].

\section{Erweiterte MRT-Bildgebung}

Eine der vielversprechendsten MRT-Technologien in Bezug auf eine frühzeitige Erkennung demenzieller Erkrankungen ist aufgrund der kurzen Akquisitionszeit das Arterial-Spin-Labelling. Nachdem erste stabile ASLSequenzen kommerziell erhältlich sind, scheint auch eine flächendeckende Verfügbarkeit gewährleistet. ASL und FDG-PET sind bezüglich der einer Atrophie vorausgehenden Hypoperfusions- und Hypometabolismusmuster durchaus vergleichbar [23]. Noch fehlen bei der ASL - im Unterschied zur FDG-PET - jedoch einfach anwendbare voxel- oder regionenbasierte Vergleiche zwischen individuellem Patient und einer Gruppe alters- und geschlechtsgleicher Gesunder.

\section{Primäre Demenz- und mit Demenz einhergehende neurodegenerative Erkrankungen}

\section{Alzheimer-Demenz}

Die ADlässt sich in ein präklinisches, ein Prodromalund ein Demenzstadium unterteilen.

Merke

Zeigen sich im präklinischen und Prodromalstadium für eine AD typische Veränderungen, gelten diese als Hinweis auf das Entstehen einer späteren Demenz

( A Abb. 2, > Abb. 3) [24].

Zu diesen Veränderungen gehören:

- erniedrigtes $A \beta_{42}$ - und erhöhtes Tau-Protein im Liquor

- temporoparietaler Hypometabolismus in der

FDG-PET
- mesiotemporale bzw. temporoparietale Atrophie in der MRT

In etwa 90\% der Fälle ist das klinische Leitsymptom die Störung des episodischen Kurzzeitgedächtnisses, in 10\% der Fälle sind die klinischen Symptome und die MRTVeränderungen verschieden:

- Bei der posterioren kortikalen Atrophie (PCA), einer atypischen AD-Form, stehen die Störung des Se-

hens, Erkennens, Handelns (Agnosie, Neglect, Apraxie) und eine damit in Verbindung zu bringende parietale Atrophie im Vordergrund.

- Die frontale Variante zeigt eine Überlappung mit der behavioralen Variante der FTD.

- Die logopenische Aphasie (LPA), eine weitere atypische AD-Form, zeigt ein links-akzentuiertes Atrophiemuster im posterioren und superioren Temporallappen sowie im inferioren Parietallappen. Die bei der LPA vorkommenden Ablagerungen von AD-typischem Amyloid- $\beta$ und FTD-typischem 3R-Tau lässt wiederum einen entitätsüberschreitenden Pathomechanismus vermuten.

\section{Vaskuläre Demenz}

Es gibt einen direkten Zusammenhang zwischen Altern, kognitiver Leistungsfähigkeit und kardiovaskulären Risikofaktoren, ferner zwischen AD und vaskulärer Demenz, sodass es häufig nicht einfach ist, vaskuläre Demenzen und Mischformen zwischen vaskulärer und $A D$ zu unterscheiden. Klinisch wird das u.a. mit dem Hachinski-Ischämie-Score versucht, in dem verschiedene, neben dem kognitiven Abbau auffällige klinische Merkmale stärker gewichtet werden. Dazu gehören abrupter Beginn, Schlaganfälle in der Anamnese und fokale neurologische Defizite; Merkmale also, die für eine vaskuläre Demenz sprechen [25]. Nach den NINDS-AIREN-Kriterien müssen für die Diagnose „vaskuläre Demenz" folgende Kriterien vorliegen:

- klinische Charakteristika einer Demenz

- klinische Charakteristika einer zerebralen Gefäßerkrankung

- zeitlicher Zusammenhang zwischen diesen beiden oder ein plötzlicher Beginn und/oder fluktuierender Verlauf der Demenz

- Bestätigung vaskulärer Läsionen durch zerebrale Bildgebung oder Histopathologie

Sowohl makro- als auch mikroangiopathische Läsionen können eine Demenz hervorrufen.

\section{Merke}

Ob sich eine Demenz entwickelt, hängt von der Lage der Läsionen und dem Schweregrad der Veränderungen ab [26]. 


\section{FALLBEISPIEL}

Ein 73-jähriger, ansonsten weitgehend gesunder Mann stellt sich in der Gedächtnis-Sprechstunde einer Universitätsklinik vor. Er fühle sich seit Jahren unsicher, erstelle aber weiterhin Gutachten als Kinder- und Jugend-Psychotherapeut. Der behandelnde Arzt diagnostiziert eine leichte kognitive Störung (ICD: F06.7) und veranlasst eine MRT. Die MRT zeigt eine mesiale Atrophie mit einem MTA-Score von 2 rechts und 3 links ( $\mathbf{A} \mathbf{b} \mathbf{b} . \mathbf{2} \mathbf{a}$ ), die intraparietalen Furchen sind links-betont erweitert, entsprechend einem Koedam-Score von mindestens 2 ( $\mathbf{A} \mathbf{A b} \mathbf{b} . \mathbf{2} \mathbf{b})$. Die volumetrische Analyse ( $\boldsymbol{A} \mathbf{A b b} . \mathbf{2} \mathbf{c})$ zeigt eine spezifische Atrophie vom mesialen Temporallappen und temporoparietalem Kortex über die in Blautönen dargestellte Abnahme des Volumens der grauen Substanz, sowie der in Rot- bis Gelbtönen dargestellten Zunahme des Volumens der entsprechenden äußeren Liquorräume. Die zusätzlich durchgeführte Support-Vektor-Maschine-Analyse ( $\mathbf{A b b}$. 2 d) zeigt eine sehr hohe Wahrscheinlichkeit für das Vorliegen einer Alzheimer-Demenz-typischen Atrophie (AD-L=AD-like). 18 Monate später hat die Atrophie deutlich zugenommen ( $\mathbf{A b b . 3 )}$, auch das klinische Bild entspricht jetzt einer AD.
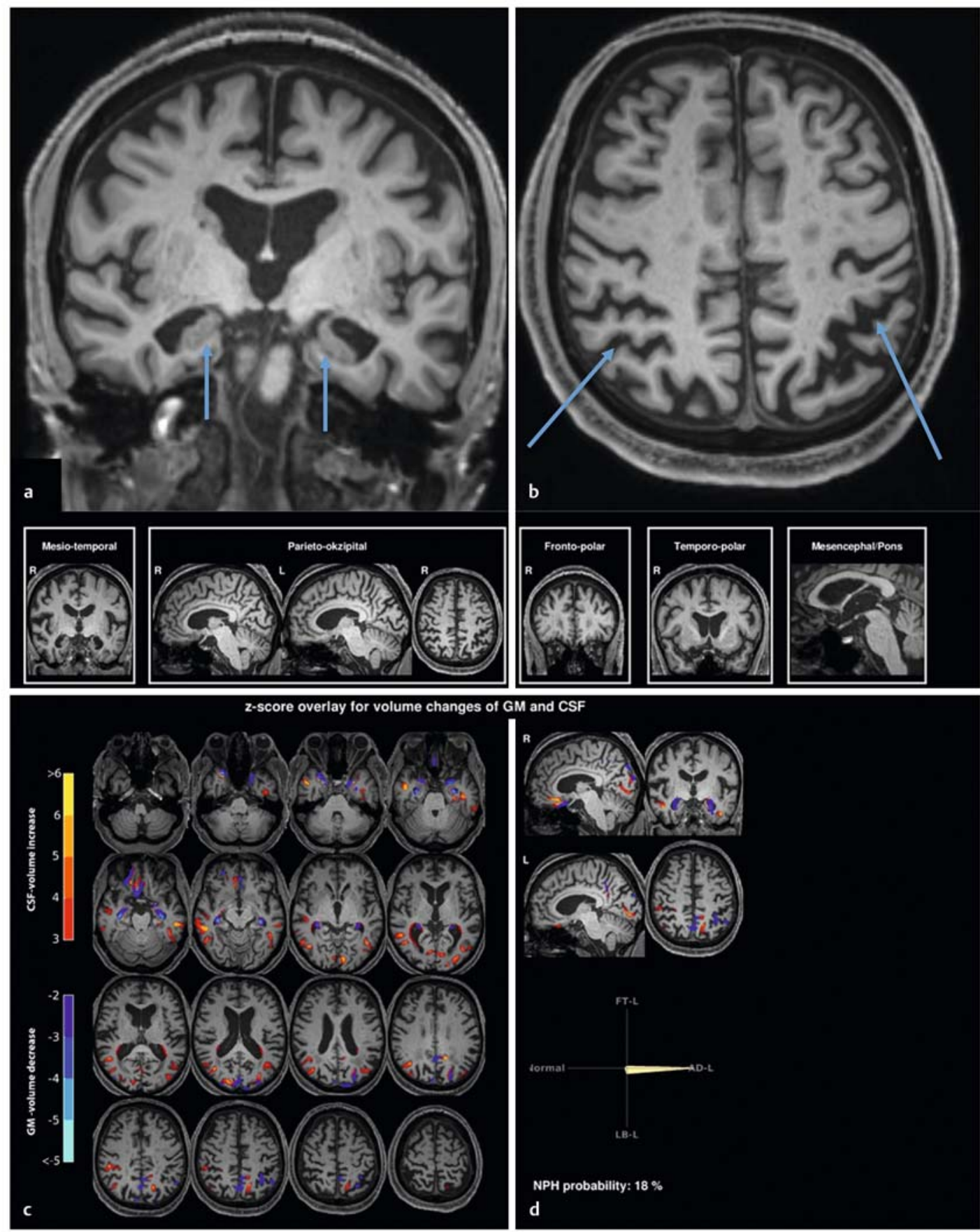

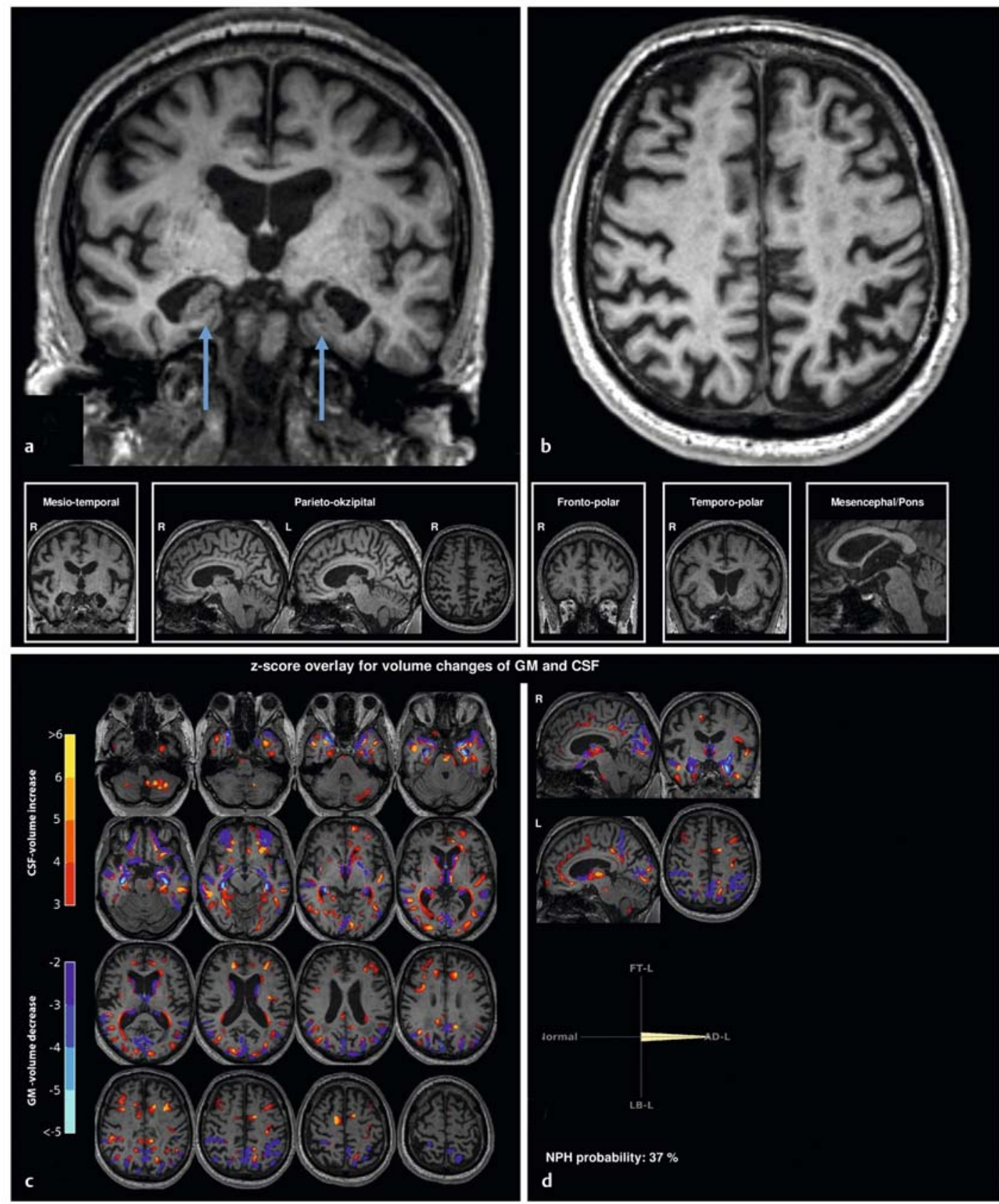

Abb. 3 Bei der Verlaufsuntersuchung nach 18 Monaten hat die Alzheimer-typische Atrophie zugenommen (Pfeile in a, b; c). Der Radarplot (d) zeigt unverändert eine hohe Wahrscheinlichkeit für ein AD-typisches Atrophiemuster (AD-L). Klinisch besteht jetzt das Bild einer Alzheimer-Demenz.

- Abb.2 (siehe gegenüberliegende Seite) 73-jähriger Mann mit leichter kognitiver Störung (ICD: F06.7). Die MRT zeigt eine mesiale Atrophie mit einem MTA-Score von 2 rechts und 3 links (a), die parietalen Furchen sind links-betont erweitert entsprechend einem Koedam-Score von 2 (b). Die volumetrische Analyse (c) stellt die Abnahme des Volumens der grauen Substanz (in Blautönen) sowie der Zunahme des Volumens der Liquorräume (in Rotbis Gelbtönen) im Vergleich zu altersund geschlechtsgematchten Gesunden dar. Der auf der Basis einer Support-Vektor-Maschine erstellte Radarplot mit den 4 Kategorien (normal = zu keiner der 3 anderen Gruppen passend, AD-L=Alzheimer's disease-like, FT-L=Fronto-temporal dementia-like und LB-L=Lewy Body dementia-like) zeigt in diesem frühen klinischen Stadium bereits eine sehr hohe Wahrscheinlichkeit für ein Alzheimer-typisches Atrophiemuster (d). 
Leukenzephalopathien werden als ausreichend für die Entwicklung einer Demenz angesehen, wenn sie mindestens ein Viertel der weißen Substanz einnehmen oder mit anderen vaskulären Läsionen gemeinsam auftreten [26].

\section{Frontotemporale Demenz}

Die FTD wird in klinisch definierte Prägnanztypen unterteilt, die im Frühstadium unterscheidbar sind, dann aber ineinander übergehen können:

- behaviorale Variante (Verhaltensvariante) der FTD (bvFTD) [27]

- primär progressive Aphasie (PPA) mit 2 FTD-Varianten (nicht flüssige PPA und semantische PPA)

- logopenische PPA (LPA), die zwischenzeitlich aufgrund der zusätzlich zur Tau-Pathologie gezeigten Amyloid- $\beta$-Komponente eher als atypische AD-Form angesehen wird [28]

Des Weiteren kann eine FTD zusammen mit einer Motoneuron-Erkrankung (FTD-MND) oder mit atypischen Parkinson-Syndromen auftreten [29].

$50 \%$ der FTD-Erkrankungen sind mit TDP43-Proteinen (FTD-TDP), $40 \%$ mit Tau-Proteinen (FTD-Tau) und 10\% mit FUS („fused in sarcoma“) (FTD-FUS) oder UbiquitinProteinen (FTD-UPS) assoziiert.

\section{Merke \\ In der MRT zeigen die klinischen Prägnanztypen der FTD entsprechende Atrophiemuster.}

Die bvFTD zeigt ein primär symmetrisches frontales Atrophiemuster mit mehr oder weniger temporaler Beteiligung ( $>$ Abb.4). Entsprechend der Klinik ist eher der nicht sprachdominante Temporallappen beteiligt. Bei der nicht flüssigen PPA ist vorwiegend das frontale Operculum betroffen. Bei der semantischen PPA ist pathognomonisch die Atrophie hauptsächlich auf den vorderen Temporallappen der sprachdominanten Hemisphäre beschränkt. Die mesiotemporalen Strukturen können dabei mitbetroffen sein ( $\mathbf{A b b}$. 5).

\section{Morbus Parkinson}

Beim durch die Trias Rigor - Tremor-Akinese charakterisierten Parkinson-Syndrom (PD) degenerieren zunächst die dopaminergen Neurone der Pars compacta der Substantia nigra. Die dopaminergen Neurone sind in sog. Nigrosomen geclustert, das größte von 5 Nigrosomen ist das Nigrosom 1 im kaudalen und posterolateralen Anteil der Substantia nigra. Die Axone der dopaminergen Neurone enden im Striatum (Putamen und Nucleus caudatus), wobei der Dopamintransport im hinteren lateralen Putamen zuerst betroffen ist. Das Dopamin(transporter)-Defizit wird mit dem ${ }^{123}$ I-FP-CIT-SPECT (DaTSCAN) mit hoher Sensitivität dargestellt.

\begin{abstract}
Merke
Es kommt nicht zur Atrophie des Gewebes, sodass morphometrische Analysen der Stammganglien sowie des Mittelhirns und der Substantia nigra beim Morbus Parkinson einen Normalbefund zeigen.
\end{abstract}

Der Verlust an dopaminergen Neuronen im Nigrosom 1 führt dazu, dass die normalerweise vorhandene Hyperintensität innerhalb der hinteren unteren Anteile der auf SWI-Aufnahmen hypointensen Substantia nigra verschwindet. Das sog. Schwalbenschwanz-Zeichen geht somit beim Morbus Parkinson verloren [30]. Eine mäßige Intrarater-Übereinstimmung sowie Unterschiede zwischen verschiedenen Geräteherstellern führt dazu, dass das Schwalbenschwanz-Zeichen in der Diagnostik nur komplementären Charakter hat.

\section{Atypische Parkinson-Syndrome}

Zu den atypischen Parkinson-Syndromen zählen die $\alpha$ Synucleinopathien LBD, MSAc, MSAp und die Tau-Erkrankungen kortikobasale Degeneration (CBD) sowie die PSP einschließlich ihrer Varianten ( $\triangleright$ Tab. 1 ).

\section{Lewy-Body-Demenz}

Die LBD ist aufgrund der klinischen Charakteristika und des raschen Verlaufs gut klinisch diagnostizierbar. Sie zeigt keine pathognomonischen MRT-Befunde, allenfalls in Spätstadien eine Atrophie des visuellen Kortex mit weiter Fissura calcarina und Sulcus parietooccipitalis.

Multisystematrophie vom zerebellären bzw. Parkinson-Prädominanztyp

Bei der MSAc findet sich eine Pons- und zerebelläre Atrophie ( $\mathbf{A b b} \mathbf{6} \mathbf{6}$ a) und auf T2w/FLAIR-Aufnahmen eine Signalerhöhung der mittleren Kleinhirnstiele sowie ein „hot cross bun“-Zeichen ( $\mathbf{A b b} \mathbf{6} \mathbf{6} \mathbf{b}$ ).

Bei der MSAp sind die hinteren Abschnitte der Putamina schlitzförmig atrophiert (,putaminal slit“) ( e), auf den $\mathrm{T} 2{ }^{*}$ - und SWI-Aufnahmen entsteht durch die vermehrte Suszeptibilität der hinteren Abschnitte

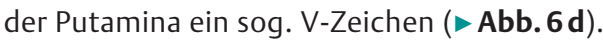

\section{Kortikobasale Degeneration}

Klinisches Leitsymptom der CBD ist das sog. „alienlimb"-Syndrom, entsprechend findet sich in der MRT eine asymmetrische Volumenminderung frontoparietal mit Betonung der Zentralregion ( $\mathbf{A b b} \mathbf{6} \mathbf{6 g}$ ).

\section{Progressive supranukleäre Paralyse}

Die PSP zeigt in der typischen Form (PSP-RS) vor allem eine Atrophie des Mittelhirns ( $\mathbf{A} \mathbf{b} \mathbf{b} . \mathbf{7} \mathbf{a}-\mathbf{c}$ ). Verschiedene planimetrische und Distanzmessungen wurden eingeführt, um diese Atrophie zu quantifizieren und die PSP gegenüber Gesunden und der MSAc abzugrenzen. Die volumetrische Analyse zeigt darüber hinaus 

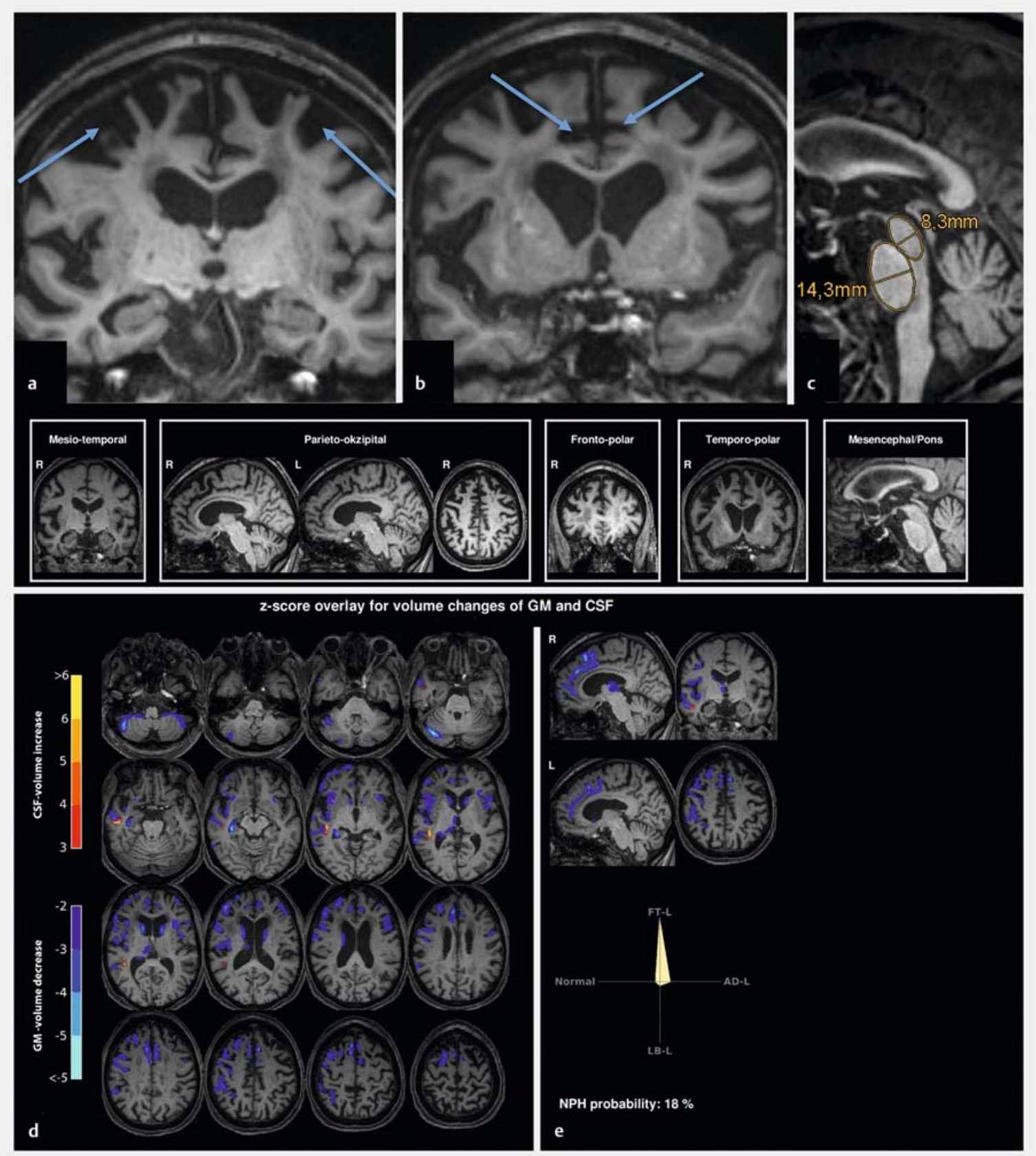

Abb. 4 71-jährige Frau mit klinisch frontalen und parietalen Defiziten. Klinisch Verdacht auf AD, DD FTD. Die MRT zeigt eine frontal betonte Erweiterung der äußeren Liquorräume (Pfeile in $\mathbf{a}$ und $\mathbf{b}$; $\mathbf{d}$ ) passend zu einer Tauopathie. Es besteht keine Mittelhirnatrophie und eine Mittelhirn-Pons-Ratio von 0,58 (c). Der Radarplot (e) zeigt eine hohe Wahrscheinlichkeit für ein FTDtypisches Atrophiemuster (FT-L). Zusätzlich Zeichen einer fortgeschrittenen Mikroangiopathie (a,b). Die MRT-morphologische Diagnose FTD wurde mittels FDG-PET bestätigt. 

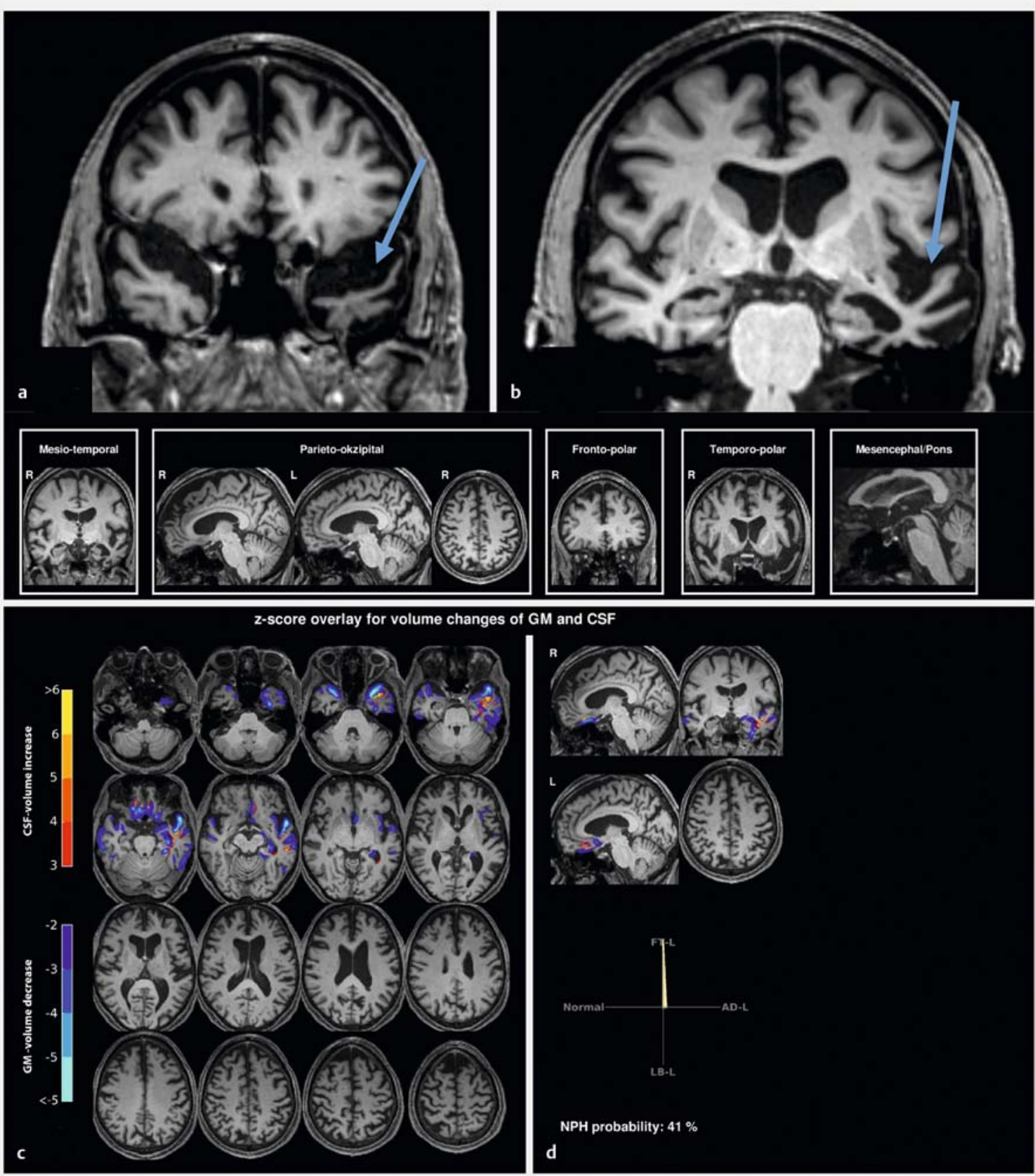

Abb. 5 77-jähriger Mann mit Wortfindungsstörungen und Neugedächtnisstörung. Der Mini-Mental Test (MMSE) mit 16 von 30 Punkten spricht für eine Demenz. Die MRT einschließlich der volumetrischen Analyse (c) zeigt eine Atrophie vor allem des vorderen und lateralen Temporallappens links (Pfeil in a und b; c), der Radarplot (d) zeigt eine hohe Wahrscheinlichkeit für eine frontotemporale Demenz (FT-L). Zusammengefasst sprechen Klinik und Atrophiemuster für eine semantische Demenz. 

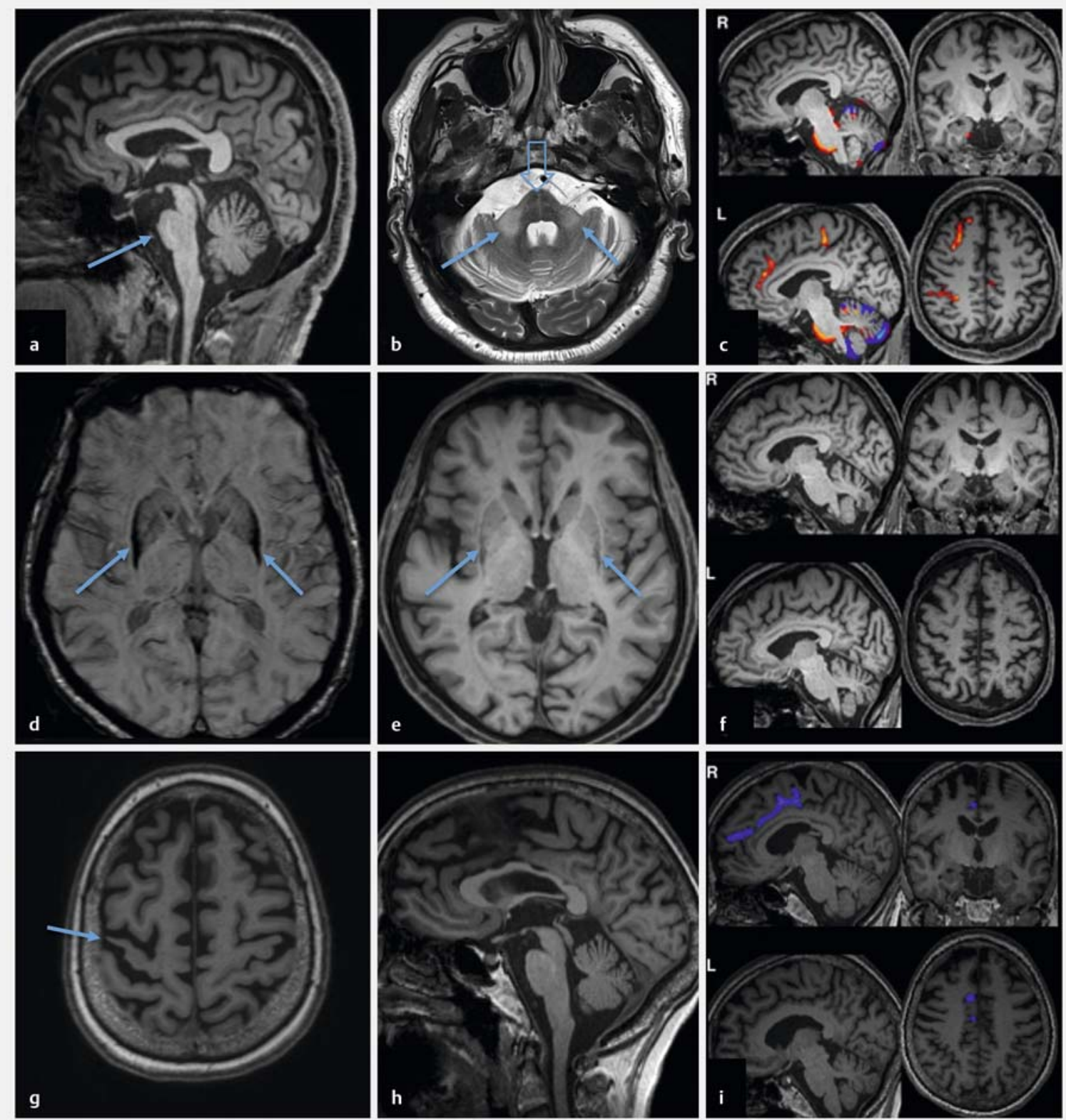

- Abb. 6 Atypische Parkinson-Syndrome: a-c MSAc mit deutlicher Pons-Atrophie (Pfeil in a), symmetrischen Signalerhöhungen der mittleren Kleinhirnstiele (Pfeile in b) und „hot cross bun“-Zeichen (offener Pfeil in b). d-f MSAp mit Atrophie und vermehrter Suszeptibilität der hinteren Putamina (Pfeile in d: SWI-Aufnahme, und e: MPRAGE-Aufnahme). Im Übrigen unauffällige Hirnvolumina (f). g-i CBD mit Erweiterung der äußeren Liquorräume mit deutlicher rechts-zentraler Atrophie (Pfeil in g). Unauffälliger Hirnstamm (h). Zusätzlich jedoch die für Tauopathien typische frontale Atrophie, ebenfalls rechts-betont (i). 

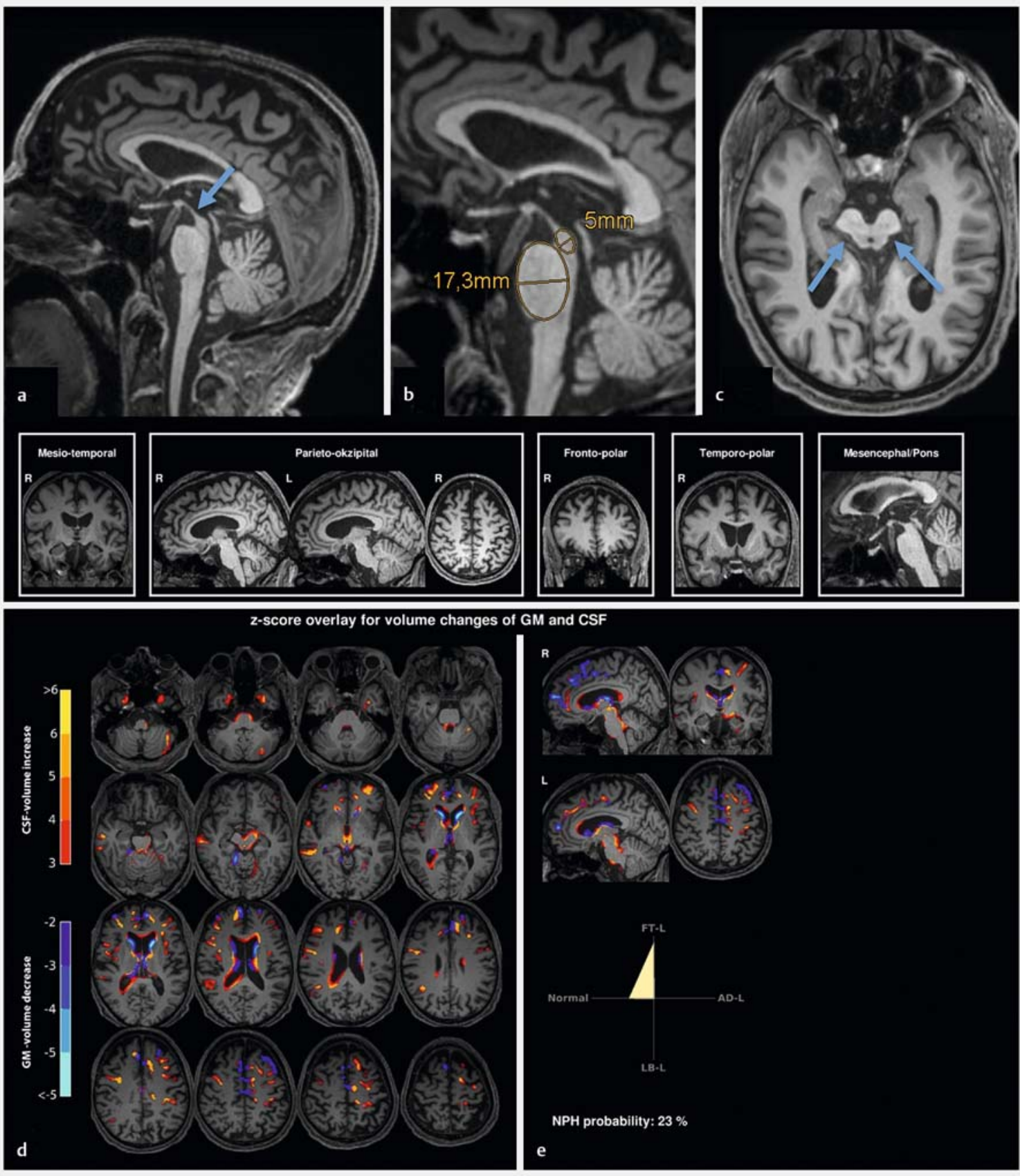

Abb.7 59-jähriger Mann mit seit 2 Jahren bestehender Bewegungsverlangsamung, unsicherem Gangbild und Verdacht auf ein atypisches Parkinsonsyndrom. Die MRT einschließlich der volumetrischen Analyse zeigt eine deutliche Mittelhirnatrophie (a-d) mit einem Kolibri-Zeichen (Pfeil in a), einer Mittelhirn-Pons-Ratio (Mittelhirn $=5 \mathrm{~mm}$, Pons $=17 \mathrm{~mm}$ ) von 0,3 (b) und einem Mickey-Mouse-Zeichen auf den axialen Aufnahmen (Pfeile in c). Neben der Mittelhirnatrophie zeigt die volumetrische Analyse (d) eine Atrophie der frontalen, vor allem frontomedialen grauen Substanz (in Blautönen) sowie eine Volumenzunahme (in Rot- bis Gelbtönen) der basalen Zisternen um das Mittelhirn (d). Entsprechend zeigt der Radarplot (e) eine hohe Wahrscheinlichkeit für ein FTD-typisches Atrophiemuster (FT-L). Das Atrophiemuster passt somit zur PSP-RS im Sinne einer Tau-Protein-Erkrankung, bei der klinisch und in der Bildgebung vornehmlich das Mittelhirn betroffen ist. 
eine frontal bis parietal betonte Hirnvolumenminderung-speziell bei den atypischen PSP-Formen [31, 32] mit teilweise geringer ausgeprägter Mittelhirnatrophie (Egger et al., unveröffentlichte Daten). Hiermit könnten unterschiedlich starke Tau-Protein-Ablagerungen und klinische PSP-Varianten erklärt werden.

\section{Fazit}

Mit Demenz einhergehende neurodegenerative Erkrankungen zeigen häufig erkrankungsspezifische Atrophiemuster. Aufgrund der starken Altersabhängigkeit der Atrophie ist die visuelle Diagnostik jedoch nicht einfach. Standardisierte Beurteilungsskalen und automatische volumetrische Analysen inkl. „machine-learning-Algorithmen“ sind dabei hilfreich.

\section{KERNAUSSAGEN}

- Mit Demenz einhergehende neurodegenerative Erkrankungen nehmen in einer älter werdenden Gesellschaft stark zu. Jeder Patient mit Verdacht auf eine solche Erkrankung sollte eine Bildgebung, idealerweise eine MRT des Kopfes erhalten.

- Mit der MRT werden in ca. $5 \%$ der Fälle behandelbare Ursachen wie Tumor, Hydrozephalus, Subduralhämatom und andere erkannt. Zudem lassen sich die weitaus häufigeren Erkrankungen Alzheimer-Demenz, vaskuläre Demenz, frontotemporale Demenz und verschiedene atypische Parkinson-Syndrome mit und ohne Demenz u. a. aufgrund ihrer regionenspezifischen Atrophiemuster unterscheiden.

- Die Erkennung der Atrophiemuster wird erleichtert, wenn der Datensatz des individuellen Patienten mit einer Gruppe „alters- und geschlechtsgematchter" Kontrollen verglichen wird und Voxel bzw. Regionen, die sich unterscheiden, hervorgehoben werden.

- Machine-Learning-Algorithmen ordnen bereits heute zuverlässig typische Atrophiemuster den entsprechenden Krankheitsentitäten zu. Mittelfristiges Ziel ist es jedoch, diese Erkrankungen nicht erst im Stadium der Atrophie zu erkennen. Eine vielversprechende Methode ist in Analogie zum FDG-PET das „Arterial Spin Labeling“, mit dem die Hypoperfusion des Hirngewebes quantitativ und regionenspezifisch dargestellt werden kann.
Interessenkonflikt

\section{Erklärung zu finanziellen Interessen}

Forschungsförderung erhalten: ja; Honorar/geldwerten Vorteil für Referententätigkeit erhalten: ja; Bezahlter Berater/interner Schulungsreferent/Gehaltsempfänger: nein; Patent/Geschäftsanteile/Aktien (Autor/Partner, Ehepartner, Kinder) an Firma (Nicht-Sponsor der Veranstaltung): nein; Patent/Geschäftsanteile/Aktien (Autor/Partner, Ehepartner, Kinder) an Firma (Sponsor der Veranstaltung): ja.

Erklärung zu nichtfinanziellen Interessen

Co-Editor Clin Neuroradiology Gesellschafter Veobrain $\mathrm{GmbH}$

\section{Autorinnen/Autoren}

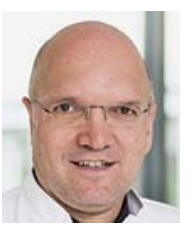

\section{Horst Urbach}

Prof. Dr. med. 1981-1988 Chemie- und Medizinstudium in Bonn. 1988 Approbation. 1989 Promotion. 1988-1995 Ausbildung zum Arzt für Radiologie und Neuroradiologie in Andernach, Koblenz und Bonn. 1994 Facharzt Radiologische Diagnostik. 1995 Schwerpunkt Neuroradiologie. 1996 Oberarzt Neuroradiologie Uniklinik Würzburg. 1997-2013 Oberarzt Neuroradiologie Uniklinik Bonn. 2000 Habilitation. Seit 2013 Ärztlicher Direktor der Klinik für Neuroradiologie, Universitätsklinik Freiburg.

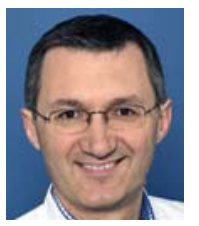

\section{Karl Egger}

PD Dr. med. 1992-1995 Studium der Betriebswirtschaftslehre in Wien und Linz. 1995-2003 Medizinstudium in Innsbruck. 2003 Promotion. 2003-2005 Wissenschaftlicher Mitarbeiter, Klinik für Neurologie, Innsbruck. 2005-2010 Assistenzarzt, Klinik für Radiologie, Innsbruck. 2010 Facharzt für Radiologie. 20102011 Research Associate, Psychiatry Neuroimaging Laboratory, Brigham and Women's Hospital, Harvard Medical School, Boston, USA. 2011-2013 Facharzt, Klinik für Neuroradiologie Freiburg, seit 2013 Oberarzt. 2013 Schwerpunktbezeichnung Neuroradiologie. 2018 Habilitation.

\section{Korrespondenzadresse}

Prof. Dr. Horst Urbach

Universitätsklinikum Freiburg

Klinik für Neuroradiologie

Breisacher Str. 64

79106 Freiburg

horst.urbach@uniklinik-freiburg.de

Wissenschaftlich verantwortlich gemäß Zertifizierungsbestimmungen

Wissenschaftlich verantwortlich gemäß Zertifizierungsbestimmungen für diesen Beitrag ist Prof. Dr. med.Horst Urbach, Freiburg. 


\section{Literatur}

[1] Deutsche Gesellschaft für Psychiatrie und Psychotherapie, Psychosomatik und Nervenheilkunde, Deutsche Gesellschaft für Neurologie. S3-Leitlinie „Demenzen“. Im Internet: https://www.awmf.org/uploads/tx_szleitlinien/038013I_S3-Demenzen-2016-07.pdf Stand: 30.04.2019

[2] Ziegler U, Doblhammer G. Prevalence and incidence of dementia in Germany - a study based on data from the public sick funds in 2002. Gesundheitswesen 2009; 71: 281-290

[3] Mackenzie IR, Rademakers R, Neumann M. TDP-43 and FUS in amyotrophic lateral sclerosis and frontotemporal dementia. Lancet Neurol 2010; 9: 995-1007

[4] Jack CRJr., Knopman DS, Jagust WJ et al. Hypothetical model of dynamic biomarkers of the Alzheimer's pathological cascade. Lancet Neurol 2010; 9: 119-128

[5] Qiu C, De Ronchi D, Fratiglioni L. The epidemiology of the dementias: an update. Curr Opin Psychiatry 2007; 20: 380385

[6] Klöppel S, Peter J, Ludl A et al. Applying Automated MR-Based Diagnostic Methods to the Memory Clinic: A Prospective Study. J Alzheimers Dis 2015; 47: 939-954

[7] Weder ND, Aziz R, Wilkins K et al. Frontotemporal dementias: a review. Ann Gen Psychiatry 2007; 6: 15

[8] Buter TC, van den Hout A, Matthews FE et al. Dementia and survival in Parkinson disease: a 12-year population study. Neurology 2008; 70: 1017-1022

[9] Zaccai J, McCracken C, Brayne C. A systematic review of prevalence and incidence studies of dementia with Lewy bodies. Age Ageing 2005; 34: 561-566

[10] Gifford DR, Hollaway RG, Vickrey BG. Systematic review of clinical prediction rules for neuroimaging in the evaluation of dementia. Arch Intern Med 2000; 160: 2855-2862

[11] Hejl A, Høgh P, Waldemar G. Potentially reversible conditions in 1000 consecutive memory clinic patients. J Neurol Neurosurg Psychiatry 2002; 73: 390-394

[12] Engel DC, Adib SD, Schuhmann MU et al. Paradigm-shift: radiological changes in the asymptomatic $\mathrm{iNPH}$-patient to be: an observational study. Fluids Barriers CNS 2018; 15: 5

[13] Ishii K, Kanda T, Harada A et al. Clinical impact of the callosal angle in the diagnosis of idiopathic normal pressure hydrocephalus. Eur Radiol 2008; 18: 2678-2683

[14] Scheltens P, Leys D, Barkhof F et al. Atrophy of medial temporal lobe on MRI in "probable" Alzheimer's disease and normal ageing: diagnostic value and neuropsychological correlates. J Neurol Neurosurg Psychiatry 1992; 55: 967972

[15] Koedam EG, Lehmann M, van der Flier WM et al. Visual assessment of posterior atrophy development of a MRI rating scale. Eur Radiol 2011; 21: 2618-2625

[16] Westman E, Cavalin L, Muehlboeck JS et al. Sensitivity and Specificity of Medial Temporal Lobe Visual Ratings and Multivariate Regional MRI Classification in Alzheimer's Disease. PLoS One 2011; 6: e22506. doi:10.1371/journal. pone.0022506

[17] Braak H, Braak E. Neuropathological stageing of Alzheimerrelated change. Acta Neuropathol 1991; 82: 239
[18] Enkirch S], Traschütz A, Müller A et al. The ERICA Score: An MR Imaging-based Visual Scoring System for the Assessment of Entorhinal Cortex Atrophy in Alzheimer Disease. Radiology 2018; 288: 226-333

[19] Massey LA, Jäger HR, Paviour DC et al. The midbrain to pons ratio: A simple and specific MRI sign of progressive supranuclear palsy. Neurology 2013; 80: 1856-1861

[20] Morelli M, Arabia G, Salsone M. Accuracy of magnetic resonance parkinsonism index for differentiation of progressive supranuclear palsy from probable or possible Parkinson disease. Mov Disord 2011; 26: 527-533

[21] Shattuck DW, Mirza M, Adisetiyo V et al. Construction of a 3D probabilistic atlas of human cortical structures. NeuroImage 2008; 39: 1064-1080

[22] Klöppel S, Yang S, Kellner E et al. Voxel-wise deviations from healthy aging for the detection of region-specific atrophy. Neuroimage Clin 2018; 20: 851-860

[23] Faellmar D, Haller S, Lilja JM et al. Arterial spin labeling-based Z-maps have high specificity and positive predictive value for neurodegenerative dementia compared to FDG-PET. Eur Radiol 2017; 27: 4237-4246

[24] Dubois B, Feldman HH, Jacova C et al. Advancing research diagnostic criteria for Alzheimer's disease: the IWG-2 criteria. Lancet Neurol 2014; 13: 614-629

[25] Hachinski VC, Iliff LD, Zilhka E et al. Cerebral blood flow in dementia. Arch Neurol 1975; 32: 632-637

[26] Roman GC, Tatemichi TK, Erkinjuntti T et al. Vascular dementia: diagnostic criteria for research studies. Report of the NINDS-AIREN International Workshop. Neurology 1993; 43: $250-260$

[27] Rascovsky K, Hodges JR, Knopman D et al. Sensitivity of revised diagnostic criteria for the behavioural variant of frontotemporal dementia. Brain 2011; 134: 2456-2477

[28] Gorno-Tempini ML, Hillis AE, Weintraub S et al. Classification of primary progressive aphasia and its variants. Neurology 2011; 76: 1006-1014

[29] Rohrer J, Warren JD. Phenotypic signatures of genetic frontotemporal dementia. Curr Opin Neurol 2011; 24: 542-549

[30] Schwarz ST, Afzal M, Morgan PS et al. The 'Swallow Tail' Appearance of the Healthy Nigrosome - A New Accurate Test of Parkinson's Disease: A Case-Control and Retrospective Cross-Sectional MRI Study at 3T. PLoS One 2014; 9: e93814. doi:10.1371/journal.pone.0093814

[31] Höglinger GU, Respondek G, Stamelou M et al. Clinical diagnosis of progressive supranuclear palsy: The movement disorder society criteria. Mov Disord 2017; 32: 853-864

[32] Sakurai K, Tokumaru AM, Shimoji K et al. Beyond the midbrain atrophy: wide spectrum of structural MRI finding in cases of pathologically proven progressive supranuclear palsy. Neuroradiology 2017; 59: 431-443

[33] McKeith IG, Boeve BF, Dickson DW et al. Diagnosis and management of dementia with Lewy bodies. Neurology 2017; 89: $88-100$

Bibliografie

DOI https://doi.org/10.1055/a-1073-2101

Neurologie up2date 2020; 3: 87-104

(c) Georg Thieme Verlag KG Stuttgart · New York

ISSN 2511-3453 


\section{Punkte sammeln auf CME.thieme.de}

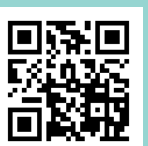

Diese Fortbildungseinheit ist in der Regel 12 Monate online für die Teilnahme verfügbar.

Den genauen Einsendeschluss finden Sie unter https://cme.thieme.de.

Sollten Sie Fragen zur Online-Teilnahme haben, finden Sie unter https://cme.thieme.de/hilfe

eine ausführliche Anleitung. Wir wünschen viel Erfolg beim Beantworten

der Fragen!

Unter https://eref.thieme.de/CXEB3V5 oder über den QR-Code kommen Sie direkt zur Startseite des Wissenstests.

VNR 2760512020158724555

\section{Frage 1}

Welche Erkrankung ist eine „behandelbare“ Demenz?
A Normaldruckhydrocephalus
B Morbus Alzheimer
C Demenz mit Lewy-Körperchen
D Creutzfeld Jakob Disease (CJD)
E Frontotemporale Lobärdegeneration (FTLD)

\section{Frage 2}

Welche Aussage zur Epidemiologie ist richtig?

A Männer erkranken häufiger als Frauen.

B Der Erkrankungsgipfel liegt im 8. Lebensjahrzehnt.

C Die Anzahl von Demenzerkrankungen wird auf 1 Mio. geschätzt.

D FTLD-Erkrankungen treten eher früher als die AlzheimerDemenz auf.

E Männer und Frauen sind in gleicher Häufigkeit betroffen.

\section{Frage 3}

Welche Erkrankung ist kein atypisches Parkinson-Syndrom?
A LBD
B PSP-RS
C PSP-CBD
D CJD
E MSAP

\section{Frage 4}

Welche Aussage zum MTA-Score ist richtig?

A Der MTA-Score beruht auf voxelbasierten Analysen.

B Der MTA-Score erfasst die Ventrikelerweiterung beim Normaldruckhydrocephalus.

C Der MTA-Score erfasst die Vergrößerung der Amygdala.

D Ein MTA-Score von 2 ist bei 70jährigen pathologisch.

E Der MTA-Score beurteilt die Erweiterung des Sulcus collateralis.

\section{Frage 5}

Welche Aussage ist richtig?

A Der Morbus Alzheimer ist durch Amyloid-Ablagerungen in den Frontallappen charakterisiert.

B Der Morbus Alzheimer beginnt im entorhinalen Kortex.

C Die PSP ist eine $\alpha$-Synuclein-Erkrankung.

D Die Erweiterung der Liquorräume um das Mittelhirn ist typisch für eine MSAc.

E Die FTLD tritt v. a. bei über 80jährigen auf.

\section{Frage 6}

Welche Zuordnung von Erkrankung und Atrophie ist richtig?
A FTLD-Parietallappen
B Morbus Alzheimer-Fontallappen
C DLB-Frontallappen
D Semantische Demenz - Temporallappen
E Morbus Alzheimer-Occipitallappen

\section{Frage 7}

Welche Aussage zum Morbus Parkinson ist richtig?
A Beim Morbus Parkinson liegt kein Schwalbenschwanz- Zeichen vor.
B Bei atypischen Parkinson-Syndromen findet sich eine Atrophie des G.präcentralis.
C Die dopaminergen Neurone befinden sich im Tegmentum mesencephali.
D Morbus Parkinson geht selten mit einer Demenz einher.
E Typische Symptome der Erkrankung sind Gangstörung und Harninkontinenz.




\section{Punkte sammeln auf CME.thieme.de}

Fortsetzung ...

\section{Frage 8}

Welche Aussage zu voxelbasierten Analysen ist richtig?

A Normalisierung heißt die Aufteilung in graue Substanz, weiße Substanz und Liquor.

B Segmentierung heißt die Transformation auf ein Standardgehirn.

C Voxelbasierte Analysen verwenden axiale DWI-Aufnahmen.

D Durch die bei der Normalisierung verwendete Modulation bleibt die Information über das ursprüngliche Volumen enthalten.

E Voxelbasierte Analysen verwenden axiale Perfusions-Aufnahmen.

\section{Frage 9}

Welche Zuordnung ist richtig?
A PSP-putaminal slit
B MSAc - hot cross bun
C PSP - eye of the tiger-Zeichen
D DLB - Koedam score
E CBD - MTA score

\section{Frage 10}

Welche Aussage zur FTLD ist richtig?

A Es handelt sich um eine Synuclein-Erkrankung.

B Typisch ist die Beteiligung der medialen Frontallappen.

C Es handelt sich häufig um eine Amyloid-Erkrankung.

D Erster Hinweis ist die Erweiterung der Fissura choroidea.

E Typisch ist die Beteiligung der medialen Parietallappen. 